All letters are subject to editing and may be shortened. General letters can be sent to bjgpdisclarcgp.org.uk (please include your postal address for publication), and letters responding directly to BJGParticles can be submitted online via eLetters. We regret we cannot notify authors regarding publication.

For submission instructions visit: bjgp.org/letters

\section{Clinical coding of long COVID in English primary care: a federated analysis of 58 million patient records in situ using OpenSAFELY}

Our paper in the $B J G P^{1}$ on diagnostic coding of long COVID described wide variation in use of the codes, and dramatically lower rates of use of the diagnostic coding when compared with long COVID as measured in self-reported survey data. As COVID is an unprecedented and evolving situation, we are providing updated analyses for key findings. A report on long COVID diagnostic code usage can be found on the OpenSAFELY reports website (reports. opensafely.org). ${ }^{2}$

As of this letter, data are current to 5 September 2021, providing 19 weeks' additional follow-up time from the original paper. During this period, 33827 additional people had a code for long COVID recorded, making 57100 people in total. The rate at which new diagnoses are being recorded remains largely unchanged. The overall prevalence of coded long COVID diagnosis in the total population is now 99.6 per 100000 , compared with 40.1 per 100000 in the original paper, due solely to greater followup time. As before, prevalence of coding in EMIS practices (126.0) remains higher than in TPP practices (63.1); however, this gap is diminishing over time, with a rate ratio of 2.0 now, and 2.6 at 19 weeks previously.

As we discussed in our article, it is critical for research and planning of services that GPs are able to appropriately code cases of long COVID. We will continue to update this report regularly to inform clinical coding of long COVID. Readers are encouraged to view the full updated report to see trends at the time of reading. ${ }^{2}$

Alex J Walker,

Epidemiologist, The DataLab, Nuffield Department of Primary Care Health Sciences, University of Oxford, Oxford. Email: alex.walkerlphc.ox.ac.uk
Brian MacKenna,

Honorary Clinical Researcher, The DataLab, Nuffield Department of Primary Care Health Sciences, University of Oxford, Oxford.

\section{Ben Goldacre,}

Director, The DataLab, Nuffield

Department of Primary Care Health

Sciences, University of Oxford, Oxford.

(On behalf of all OpenSAFELY study authors.)

\section{REFERENCES}

1. Walker AJ, MacKenna B, Inglesby P, et al. Clinical coding of long COVID in English primary care: a federated analysis of 58 million patient records in situ using OpenSAFELY. Br J Gen Pract 2021; DOI: https://doi.org/10.3399/BJGP.2021.0301.

2. Walker AJ, MacKenna B, Inglesby P, et al. Clinical coding of long COVID in English primary care: a federated analysis of 58 million patient records in situ using OpenSAFELY. 2021; DOI: http://doi. org/10.53764/rpt.3917ab5ac5.

DOI: https://doi.org/10.3399/bjgp21X717449

\section{Interpretation of ethnicity-specific data: increased risk versus increased utilisation}

It was with great pleasure we read the article by Robson et al titled 'NHS Health Checks: an observational study of equity and outcomes 2009-2017.' We would like to offer additional contributions regarding explanations for the findings and differences between ethnic groups. It is difficult to ascertain whether ethnic disparities in incidence of disease between attendees and non-attendees are due to underlying higher risk of disease in these groups or the result of the NHS Health Check. It is well established that Black and South Asian patients have increased risk of hypertension and diabetes compared with White patients, and that ethnicity- specific body mass index (BMI) cutoffs should be utilised. It would be important to understand the risk of incident disease in attendees versus non-attendees within each ethnic group.

Interestingly, in a recent 2021 Lancet article, study authors found that adjusted incidence of type 2 diabetes occurred at far lower BMI in South Asians (BMI of 23.9) and Black Caribbeans (BMI of 26.0) compared with White patients with BMI of 30.0.2 Additionally, at comparable BMls, Bangladeshis had the highest risk of type 2 diabetes, followed by Pakistanis and Indians. This is in line with the ethnic differences in NHS Health Check attendance rates, potentially offering an explanation for South Asians' high attendance rates.

Lastly, Eastwood et al, in a June 2021 article assessing UK ethnic differences in statin initiation, found that time to initiation of statins was longest for South Asians, followed by Black patients. ${ }^{3}$ They also found that South Asians and Black patients were significantly less likely to initiate statins compared with European patients. ${ }^{3}$ This disparity in the overall primary care setting may overestimate the ethnic differences seen in attendees versus non-attendees. Overall, we believe the authors of this paper make a strong case regarding the low uptake and effectiveness of the NHS Health Checks and that more targeted, culturally sensitive cost-effective approaches should be considered. Further studies should keep in mind the different comorbid risk factors as well as changing national guidelines and ethnicity-specific guidance that may influence findings.

Alizah Ali,

MD Student Researcher, Morehouse School of Medicine, Atlanta, Georgia. Email: aialiamsm.edu

Fariha Hameed,

MD Student Researcher, McGovern Medical School, Houston, Texas.

Bharath Nagaraj,

MD Student Researcher, Rutgers New Jersey Medical School, Newark, New Jersey; North American Disease Intervention, New Brunswick, New Jersey. 
Aayush Visaria,

Postdoctoral Research Fellow, Rutgers University, New Brunswick, New Jersey; North American Disease Intervention, New Brunswick, New Jersey.

\section{REFERENCES}

1. Robson J, Garriga C, Coupland C, Hippisley-Cox J. NHS Health Checks: an observational study of equity and outcomes 2009-2017. Br J Gen Pract 2021; DOI: https://doi.org/10.3399/BJGP.2020.1021.

2. Caleyachetty R, Barber TM, Mohammed NI, et al. Ethnicity-specific BMI cutoffs for obesity based on type 2 diabetes risk in England: a population-based cohort study. Lancet Diabetes Endocrinol 2021. 9(7): 419-426.

3. Eastwood SV, Mathur R, Sattar N, et al. Ethnic differences in guideline-indicated statin initiation for people with type 2 diabetes in UK primary care 2006-2019: a cohort study. PLoS Med 2021; 18(6): e1003672.

\section{Primary care clinical pharmacists and chronic disease medication adherence}

The NHS Long Term Plan emphasises the vital role of prevention in the NHS of the future.' Optimising patients' medical management of chronic disease is an opportunity for primary care. Primary Care Network-based Population Health Management interventions have the potential to augment existing services. Pharmacist-led interventions to improve chronic disease medication adherence have been shown to be effective. ${ }^{2-4}$ A study of the New Medicine Service offered by community pharmacies in England showed $70 \%$ selfreported medication adherence at 10 weeks in the intervention group compared with $60 \%$ in the control. ${ }^{5}$

A Quality Improvement Project in a socioeconomically deprived general practice in Nottinghamshire led to a clinical pharmacist telephoning 30 patients with suboptimally managed lipid profiles and cardiovascular risk. Fourteen patients were prescribed and repeatedly dispensed simvastatin, atorvastatin, or rosuvastatin. Eight (57\%) of those confided in the clinical pharmacist that they were non-adherent with the lipid-lowering medication regime.
Patients had recently consulted with GPs and nurses prior to the intervention. Clinical pharmacist consultation may lead to more candid discussions about medication. A study of primary care in rural Australia ${ }^{6}$ reported a similar finding. Clinical pharmacists asked 50 patients about their drug history. Forty per cent of patients reported they were not adherent to their prescribed medication regime.

All but one of the eight non-adherent Nottinghamshire patients agreed to restart medication after consultation. The most given reason for non-adherence was not feeling any benefit from taking pills regularly. The pharmacist reflected that most patients had a weak understanding of the primary or secondary prevention rationale for treatment. A 2016 metaanalysis found health literacy to be positively correlated with medication adherence and that intervention can increase both. The effect of intervention was more pronounced in patients with lower incomes.

Selective intervention by clinical pharmacists may add health benefit above usual care by increasing adherence to longterm medication regimes. Low levels of health literacy in areas of socioeconomic deprivation may be a factor amenable to pharmacist intervention. Patients with greater socioeconomic deprivation and markers of poor disease control such as lipid profiles, $\mathrm{HbA} 1 \mathrm{C}$, and blood pressure could be prioritised inside a Primary Care Network footprint to maximise health gain.

Stephen Wormall,

(NIHR) In-Practice Fellow in Primary Care; GP, Clinical Design Authority, Nottingham and Nottinghamshire CCG; University of Nottingham, Nottingham.

Email: stephen.wormallanottingham.ac.uk

Sarah Abbot,

Clinical Pharmacist; Primary Integrated Care Services, Nottingham.

\section{Competing interests}

Stephen Wormall is an In-Practice Fellow supported by the Department of Health and Social Care and the National Institute for Health Research (NIHR). The views expressed are those of the author(s) and not necessarily those of the NHS, the NIHR, or the Department of Health.

\section{REFERENCES}

1. NHS. The NHS Long Term Plan. 2019. https:// www.longtermplan.nhs.uk/ laccessed 6 Oct 2021).
2. Ali F, Laurin M-Y, Lairière $C$, et al. The effect of pharmacist intervention and patient education on lipid-lowering medication compliance and plasma cholesterol levels. Can J Clin Pharmacol 2003; 10(3): 101-106.

3. Milosavljevic A, Aspden T, Harrison J. Community pharmacist-led interventions and their impact on patients' medication adherence and other health outcomes: a systematic review. Int J Pharm Pract 2018; 26(5): 387-397.

4. Elnaem MH, Rosley NFF, Alhifany AA, et al. Impact of pharmacist-led interventions on medication adherence and clinical outcomes in patients with hypertension and hyperlipidemia: a scoping review of published literature. J Multidiscip Healthc 2020; 13: $635-645$.

5. Elliott RA, Boyd M, Salmea N-E, et al. Supporting adherence for people starting a new medication for a long-term condition through community pharmacies: a pragmatic randomised controlled trial of the New Medicine Service. BMJ Qual Saf 2016; 25(10): 747-758.

6. Bonner CJ, Carr B. Medication compliance problems in general practice: detection and intervention by pharmacists and doctors. Aust $J$ Rural Health 2002; 10(1): 33-38.

7. Miller TA. Health literacy and adherence to medical treatment in chronic and acute illness: a meta-analysis. Patient Educ Couns 2016; 99(7): 1079-1086 